SOCS3 is limited by its short biological half-life. Therefore, mutation of all 9 Lys residues that are potential sites of ubiquitination to Arg should produce a mutated SOCS3 resistant to ubiquitin-mediated proteasomal degradation ("Lys-less" SOCS3). We hypothesise that a stablised "Lys-less" SOCS3 may have greater therapeutic potential versus wild type (WT) SOCS3 in limiting JAK/STAT mediated processes responsible for neointimal hyperplasia and vein graft failure in type 2 diabetes mellitus (T2DM).

Methods Smooth muscle cells (SMCs) and endothelial cell (ECs) isolated from human saphenous vein (HSV) were transduced with recombinant lentiviruses, $\mathrm{MOI}=3.6$ (WT), 22.2 (Lys-less SOCS3) and 5.6 (GFP) tu/cell. Successful transduction was confirmed by immunofluorescence and immunoblotting. Ubiquitylation was tested by immunoprecipitation and immunoblotting and half-life was determined by immunoblotting following incubation \pm protein synthesis inhibitor emetine. HSVSMC proliferation (cell counting and CyQuant proliferation assay) and migration (Boyden chamber assay) were assessed in transduced HSVSMCs. Finally, the effect of WT and Lys-less SOCS3 gene delivery on IL-6 and PDGF-BB signalling in HSVSMCs was assessed by phosphorylation of STAT3 (Tyr705) and ERK1/ 2 (Thr202/Tyr204) by immunoblotting.

Results Lentiviral transduction of WT and Lys-less SOCS3 in HSVSMCs and ECs was highly efficient after 48hrs with $97 \pm 0.9 \%(n=4)$ and was sustained for at-least 2 weeks. Lysless SOCS3 was resistant to ubiquitylation in HSVECs in contrast to WT $(n=3)$. Lys-less SOCS3 was also more stable $(\mathrm{t} 1 /$ $2=4 \mathrm{~h})$ than WT $(\mathrm{t} 1 / 2<4 \mathrm{~h})(\mathrm{n}=6, \mathrm{p}<0.001)$. Concomitant with a significant reduction in proliferative response to sIL6R $\alpha /$ IL-6 in HSVSMCs treated with WT and Lys-less SOCS3 was a selective inhibition of sIL-6R $\alpha /$ IL-6-mediated STAT3 activation by $74 \pm 6 \%$ and $80 \pm 6 \%$ respectively $(n=5, p<0.001$ versus sIL-6R $\alpha / \mathrm{IL}-6$ alone) but not ERK1/2. Time course experiments indicated that PDGF-BB-induced STAT3 and not ERK1/2 activation was blocked by WT SOCS3 in HSVSMCs by $59 \pm 4 \%$ at 5 minutes and $38 \pm 1 \%$ at 15 minutes $(n=3$ $\mathrm{p}<0.05$ versus PDGF-BB/GFP). WT and Lys-less SOCS3 did not affect proliferative responses to $20 \%$ foetal bovine serum as well as PDGF-BB-induced migration.

Conclusion WT and Lys-less SOCS3 can be successfully transduced into HSVSMCs and ECs with high efficiency using recombinant lentiviruses. Lys-less SOCS3 is more stable than WT yet functionally equivalent in inhibiting HSVSMC proliferation. WT SOCS3 was also capable of inhibiting both IL-6 and PDGF-BB signalling. These results provide evidence for the possible therapeutic targeting of SOCS3 to limit SMC dysfunction responsible for graft failure.

Conflict of interest None

\section{BS30 OUTLINING THE HUMAN MONOCYTE INFLAMMATORY CYTOKINE RESPONSE TO DIETARY FAT INTAKE}

${ }^{1}$ Mohammed Shamim Rahman*, 'Panagiotis Vorkas, 'Gary Frost, ${ }^{2}$ Douglas Morrison, ${ }^{3}$ Dorian Haskard, ${ }^{1}$ Kevin I Woollard. ${ }^{1}$ Imperial College London; ${ }^{2}$ SUERC; ${ }^{3}$ NIHR Imperial College London BRC and Imperial College Healthcare NHS Trust

10.1136/heartjnl-2019-BCS.193
Introduction Dysregulated immune homeostasis is implicated in atherosclerosis. Monocytes are described as inert innate immune cells that respond to vascular and inflammatory cues to extravasate into the intima then differentiate into 'active' macrophage foam cells on encountering lipid, resulting in the formation of fatty streaks in atherosclerotic plaque. Monocytes are however heterogeneous cells with distinct subsets; classical (CD14+CD16-) "inflammatory", non-classical (CD14-CD16+) "patrolling" and intermediate $(\mathrm{CD} 14+\mathrm{CD} 16+)$ monocytes. These subsets differ in both morphology and functional responses. Dietary saturated fat (SF) intake is somewhat controversially implicated in cardiovascular disease (CVD) from epidemiological observational and interventional studies, whilst cellular data suggest SF to provide a pro-inflammatory stimulus to leukocytes. Recent dietary and mendelian randomisation studies record a positive correlation between triglyceride rich lipoprotein (TGRL), remnant cholesterol and CVD, renewing interest in diet lipid intake as a CVD risk factor. Little is known however about the cellular nature of this risk and what role if any the innate immune system plays in inflammation and lipid trafficking. I investigated the monocyte subset response to acute dietary lipid intake intake in this context.

Methods I conducted a dietary interventional study in 8 healthy adult human volunteers fed a lauric acid-rich SF meal. Monocytes were purified from whole blood into CD16- (classical) and CD16+ (non-classical \& intermediate) subsets in the fasting and 4-hour postprandial state. Surface protein expression, cytokine release, gene expression, lipid uptake and functional responses were recorded.

Results The SF meal elicited an acute rise in serum TG in postprandial blood (fig 1A). Postprandial CD16- monocytes demonstrated an increase in HLA-DR surface protein expression (fig 1B) and preferentially accumulated intracellular neutral lipid droplets that were larger and more numerate than their CD16 + counterparts (fig 1C). Postprandial monocytes became "too fat to move" with reduced chemokinesis (fig 1D). Stable isotope tracer (13C-palmitate) combined with the SF meal confirmed exogenous dietary lipid entry into monocyte subsets (fig 1E). Postprandial monocytes downregulated inflammatory genes (fig 1F) and demonstrated an immunosuppressed response to TLR-agonist stimulation with lipopolysaccharide (LPS), further accentuated by postprandial TGRL supplementation (fig 1G). Lipidomic analysis revealed intracellular accumulation of docosahexaenoic acid (DHA) isomer 13-HODE (fig 1H) that has strong anti-inflammatory properties and a reduction in inflammatory leukotrienes (LTE4 \& LTB4), both necessary for effective TLR cytokine responses (data not shown).

Conclusion Although published data suggest SF exposure confers a pro-inflammatory phenotype to immune cells, much of this data is derived from animal models or immortalised cell lines in vitro. I have demonstrated a novel immunoparetic and perturbed chemokine response in monocytes following acute SF ingestion, with intracellular accumulation of DHA and reduction in leukotrienes the likely mechanism. CD16low monocytes preferentially accumulate cytoplasmic lipid droplets in response to dietary SF, which may be representative of lipidomic and functional changes described. This alters our current understanding of lipid and innate immune cell trafficking in the context of atherogenesis.

Conflict of interest None 